\title{
EchoGéo
}

\section{Production et usage des ressources urbaines en migration : les ateliers de confection à São Paulo}

\author{
Sylvain Souchaud
}

\section{(2) OpenEdition \\ 12 Journals}

\section{Electronic version}

URL: https://journals.openedition.org/echogeo/14828

DOI: 10.4000/echogeo.14828

ISSN: 1963-1197

\section{Publisher}

Pôle de recherche pour l'organisation et la diffusion de l'information géographique (CNRS UMR 8586)

\section{Electronic reference}

Sylvain Souchaud, "Production et usage des ressources urbaines en migration : les ateliers de confection à São Paulo", EchoGéo [Online], 39 | 2017, Online since 28 March 2017, connection on 11 August 2021. URL: http://journals.openedition.org/echogeo/14828 ; DOl: https://doi.org/10.4000/ echogeo. 14828

\section{This text was automatically generated on 11 August 2021}

EchoGéo est mis à disposition selon les termes de la licence Creative Commons Attribution - Pas d'Utilisation Commerciale - Pas de Modification 4.0 International (CC BY-NC-ND) 


\title{
Production et usage des ressources urbaines en migration : les ateliers de confection à São Paulo
}

\author{
Sylvain Souchaud
}

\section{Introduction}

1 L'industrie de la confection est un secteur d'activité important au Brésil, bien que sa production soit essentiellement destinée au marché intérieur. Aujourd'hui encore, São Paulo est le principal lieu de production et de commerce du vêtement du pays. En 2010, selon le recensement de la population (Ibge, 2012), la région métropolitaine de São Paulo (RMSP) comptait 185000 actifs dans le secteur de la confection, dont $76 \%$ de femmes, souvent originaires des États de la région Nord-Est ${ }^{1}$. Mais, depuis une vingtaine d'années, à São Paulo, la présence étrangère dans le secteur augmente et il est probable que l'immigration internationale totalise actuellement entre un tiers et la moitié des actifs de la confection ${ }^{2}$. Les immigrations bolivienne et paraguayenne occupent une place privilégiée dans l'industrie du vêtement qui est un débouché de première importance pour ces migrants, puisque en 2010 (Ibge, 2012), $63 \%$ de l'ensemble des actifs immigrés boliviens et $45 \%$ de l'ensemble des actifs immigrés paraguayens à São Paulo (RMSP) travaillent dans l'industrie de la confection, signe d'une concentration sectorielle, d'ailleurs plus marquée parmi les migrants récents. De plus, le recensement de la population de 2010 montre qu'au cours de la décennie précédente, l'immigration au Brésil a pour origines principales la Bolivie et le Paraguay, et pour destination première la région métropolitaine de São Paulo. Autant dire que l'activité de la confection est articulée au regain migratoire que connaît le pays depuis une vingtaine d'années (Souchaud, 2012). Enfin, la migration se distingue par une surreprésentation dans les ateliers de confection, qui sont l'une des trois modalités de la production de vêtements, avec la fabrication en usine et à domicile. L'ensemble de ce dispositif productif fonctionne en complémentarité, grâce aux mécanismes complexes de la sous-traitance (Bonacich et Appelbaum, 2000; Green, 
2003 ; Leite, 2004 ; Silva, 2008). Les années 1980-1990 ont vu l'activité en usine reculer, au bénéfice de la production en atelier (Pochmann, 2004) et à domicile (Buechler, 2014). Ce transfert de l'activité, de l'usine vers les ateliers, est l'illustration de la flexibilisation de l'économie brésilienne, qui intervient pendant une période de profonde crise économique et sociale, et coïncide, dans la confection, avec de nouvelles vagues d'immigration coréenne qui, installées dans le quartier de Bom Retiro à São Paulo, prennent position dans le secteur et relancent la production en atelier (Choi, 1991).

2 Aujourd'hui, la migration internationale se concentre dans les ateliers, et il est probable que l'essentiel des ateliers recourt majoritairement à la main-d'œuvre immigrée (illustration 1).

Illustration 1 - Vue générale d'un atelier du centre-ville de São Paulo, Bom Retiro

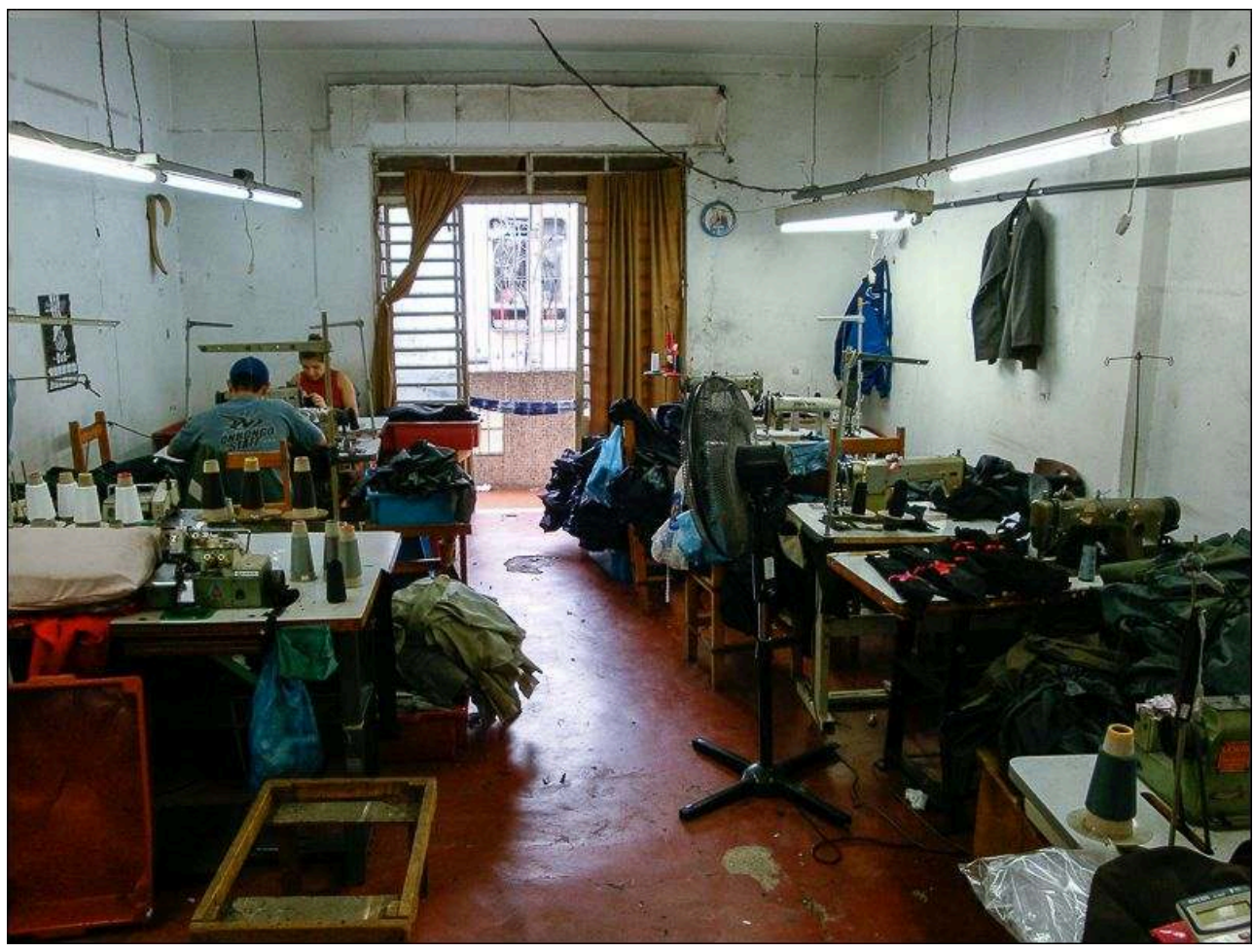

Auteur: S. Souchaud, 26 avril 2010.

3 Selon leurs observations faites aux États-Unis, les sociologues R. Waldinger et M. Lapp (Waldinger et Lapp, 1993) définissent l'atelier de confection comme une entreprise de faible envergure ("small scale»), facilement accessible (" ease of entry») car peu contraignante en termes d'investissement et d'infrastructures, où la main-d'œuvre comparée au capital constitue l'enjeu économique essentiel («labour intensiveness ») et où les arrangements informels sont multiples (« evasion of government regulation »). Cette définition correspond à ce que l'on peut observer à São Paulo, où l'atelier moyen est une structure sommaire qui compte une dizaine d'ouvriers et où les irrégularités sont nombreuses (Kontic, 2007), qu'il s'agisse des autorisations d'activité (alvará), des déclarations de production (Registre des entreprises: Cadastro nacional de pessoas jurídicas - CNPJ), de la couverture sociale de la main-d'œuvre (carteira de trabalho) ou de sa situation administrative s'agissant de migrants internationaux. 
4 Ainsi, il existe une spécificité de l'atelier de confection, en tant que dispositif à la fois économique et social qui, comme nous allons le voir, s'organise grâce à des interactions avec l'espace urbain. Notre objectif est de montrer en quoi l'atelier de confection constitue une ressource pour la migration, et comment le dispositif s'articule à des ressources urbaines, dont l'appropriation détermine en partie ses caractéristiques. Pour les migrants, les ateliers de confection sont une ressource en termes d'emploi et de logement. Par ailleurs, les ateliers sont organisés autour de la mise à profit de ressources urbaines, telles que la centralité métropolitaine et ses économies d'agglomération ou plus simplement l'offre immobilière bon marché des bâtiments sous-occupés.

Dans une première partie, nous explorerons le lien entre la migration et les ressources urbaines. Puis, nous définirons le dispositif de l'atelier de confection comme une structure reposant sur l'informalité qui associe un lieu de travail et un espace résidentiel en vue de capter la main-d'œuvre et d'optimiser sa force de travail. Nous montrerons ensuite comment la morphologie du centre-ville constitue une ressource particulière pour les ateliers de confection, en insistant notamment sur les usages mixtes du bâti qui inscrivent les ateliers dans le tissu du centre-ville. L'usage de cette morphologie peut ainsi être analysé comme une ressource urbaine, dont nous verrons pour conclure qu'il explique en partie l'informalité des ateliers.

6 Les éléments exposés dans ce texte sont le fruit d'une recherche menée entre 2007 et 2012. Elle associe une enquête de terrain, réalisée pour l'essentiel de septembre 2009 à août 2010 en fin d'un séjour de plusieurs années à São Paulo, et le traitement de données statistiques tirées principalement de la base des données individuelles du recensement de la population de 2010 (IBGE 2012). L'enquête de terrain s'est structurée autour d'observations et de relevés, et d'entretiens. Les entretiens enregistrés, conduits en espagnol avec 26 migrants boliviens et paraguayens, ont été réalisés sur le lieu de travail ou d'hébergement, et parfois lors de rendez-vous fixés dans des lieux publics. Ils nous ont servi à explorer les trajectoires migratoires, résidentielles et professionnelles et ont été complétés par des conversations informelles. Les observations et relevés ont porté sur l'organisation et l'usage fonctionnels du quartier d'étude du centre-ville, en majeure partie situé dans les districts de Bom Retiro, Brás et Pari (voir illustrations 2 et $3)^{3}$. Les informations qualitatives (observations et entretiens) sur les quartiers périphériques ont été rassemblées au cours de sorties d'une journée, à Tiradentes et Guarulhos (Vila Any). Les données des recensements de la population de 2000 et 2010 nous ont permis de décrire plus systématiquement les populations migrantes, dans le travail, le logement ou les parcours migratoires. Elles ont été complétées par les statistiques économiques ou les enquêtes de population annuelles, plus à des fins de vérifications ou de cadrages métropolitains et nationaux que d'analyse, en raison des insuffisances propres à ces données, comme le manque de précision géographique ou la non prise en compte des activités des entreprises non-déclarées ou de moins de 5 employés ${ }^{4}$. 
Illustration 2 - Localisation du centre-ville de São Paulo

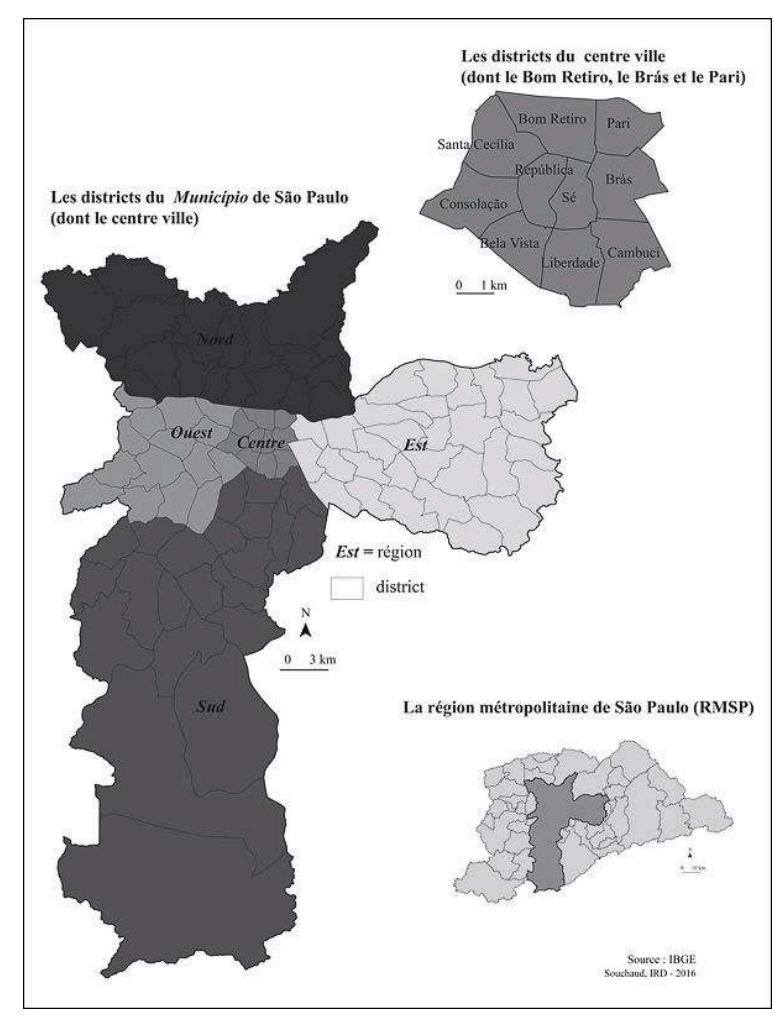

\section{Migration et ressources urbaines}

7 Pour aborder la question des ressources urbaines et sa définition, il faut dans un premier temps replacer cette notion de ressources dans le contexte de la migration urbaine.

8 À côté des déterminants classiques que sont les différentiels de revenus ou de qualification, la migration se structure grâce à la dynamique des réseaux sociaux ${ }^{5}$, propres à la migration, déployés sur l'ensemble des espaces parcourus par les migrants. Dans les lieux d'installation, les réseaux migratoires (Massey et al., 1998 ; Portes, 1995) conditionnent entre autres l'accès au travail, à un hébergement, et plus généralement aux potentialités de l'environnement local. En effet, la migration se construit grâce à la mobilisation de ressources constitutives de chacun des lieux parcourus, qu'il s'agisse des lieux d'origine, de transit ou d'installation. C'est dans la mobilité et par l'articulation des lieux et de leurs ressources que s'élaborent les trajectoires sociales des migrants. Or, leur situation socio-économique et leur statut d'étranger les placent en position de vulnérabilité et, très souvent, ils doivent faire face à une pauvreté aggravée par un statut administratif précaire et de faibles niveaux d'instruction. Ces caractéristiques accentuent l'isolement dans la société d'accueil, particulièrement important chez les primo-arrivants dont l'accès au marché du travail est très limité. Enfin, les migrants sont aussi privés d'une partie de l'encadrement de l'État et de ses ressources, parce qu'ils sont sans titre de séjour ou manquent des informations indispensables pour y prétendre. 
Illustration 3 - Le quartier de la confection dans le centre de São Paulo

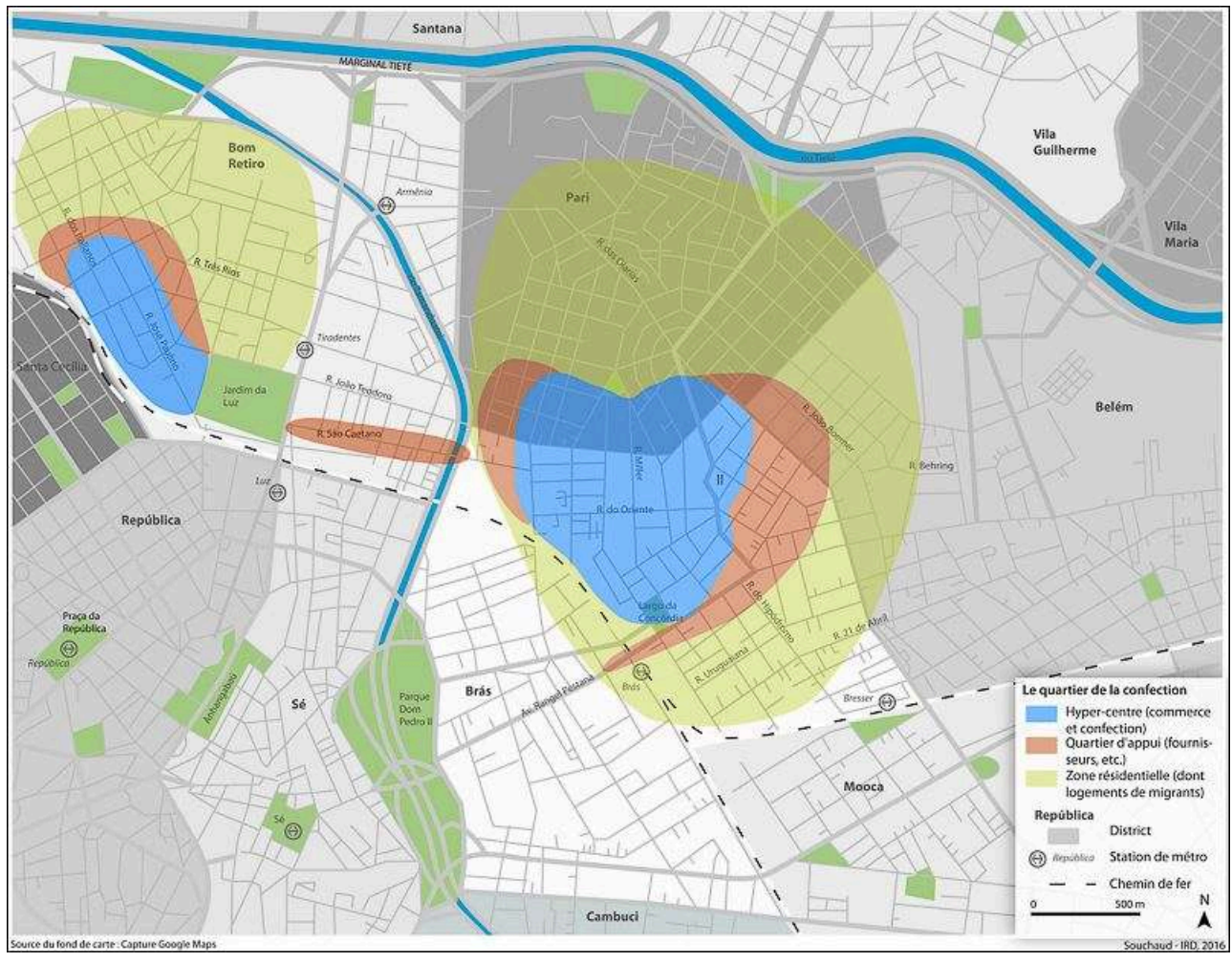

9 À São Paulo, une bonne partie de la migration récente doit faire face à cette situation. Il est ainsi très difficile, notamment pour les migrants boliviens et paraguayens, d'accéder à un emploi, même faiblement rémunéré ; tout comme il s'avère presque impossible de trouver un logement dans les circuits formels et au prix du marché. L'exclusion du marché de l'emploi et les difficultés d'accès à des ressources essentielles, comme le logement, accroissent le recours aux réseaux sociaux (Bastia, 2007 ; Light, 2002) et conduisent à la formation de niches économiques (Waldinger, 1994), comme celle de la confection en atelier. Mais elle entraîne également la nécessité de mobiliser des ressources spécifiques dans les espaces urbains qui constituent le cadre de vie des migrants : ceux-ci sont d'autant plus contraints de mobiliser les ressources du lieu qu'ils en sont individuellement dépourvus en tant que migrants et étrangers.

L'expérience urbaine des primo-arrivants est donc paradoxale. Car la ville est un lieu où ils font l'expérience de l'exclusion, mais c'est aussi l'endroit où ils espèrent trouver les ressources nécessaires à la réalisation de leur projet migratoire qui vise l'ascension sociale sur place et la réalisation d'investissements principalement dans le lieu de départ (achat d'une terre, d'un commerce, construction d'une résidence, financement d'études, etc.).

Par conséquent, en fonction de leur condition, les migrants doivent multiplier les interactions sociales et spatiales pour mobiliser des ressources souvent spécifiques. Ils changent de lieu de résidence ou d'activité, multiplient les expériences en développant différentes formes de socialisation. Ils ont donc des trajectoires sociales et spatiales complexes et spécifiques, leurs parcours sont souvent denses, mais très ancrés dans l'environnement urbain. Le migrant est ainsi un acteur urbain qui se définit dans son rapport aux ressources urbaines, qu'il mobilise et qu'il produit (Piché, 2013). 

sociale, et elles s'inscrivent dans un processus de production. En attachant la ressource à un territoire, on se rapproche en partie de la définition classique de la ressource selon laquelle elle est un caractère propre à un territoire, dont on peut tirer profit moyennant son exploitation, et qui en définit la richesse (Lamara, 2009). Les ressources ainsi définies, dites "naturelles ", sont endogènes, exploitables, elles ont un prix et elles existent en quantités finies ou non (elles sont renouvelables ou non renouvelables). Ces caractéristiques de la ressource sont également attachées à la catégorie des aménités (Cavailhès et al., 2002), notion qui regroupe les qualités données ou construites d'un environnement (variété des commerces et des services, offres culturelles et de loisirs, espaces verts, etc.), très souvent urbain, et qui intègre l'analyse des choix résidentiels et des mobilités des habitants. Suivant une approche semblable, et à partir du traitement de la Base permanente d'équipements (BPE) de l'INSEE ${ }^{6}$, J. Vallée, H. Commenges et al. (2015), définissent les ressources urbaines comme des équipements liés à des activités relatives au travail, au logement ou aux loisirs, qu'ils différencient selon qu'elles sont de proximité, intermédiaires ou supérieures (par exemple, école primaire, collège ou lycée).

13 Mais, à la différence de l'approche classique de la ressource territoriale qui est empreinte de déterminisme naturel - où la ressource, endogène, est une réalité d'un lieu -, la ressource urbaine, telle que nous l'envisageons, est associée à une production sociale: elle résulte d'un processus social qui l'inscrit dans une configuration territoriale, la ville. C'est d'ailleurs en des termes proches que Lévy et Lussault, sans mentionner un environnement particulier, définissent la ressource qui, selon eux, est « une réalité entrant dans un processus de production et incorporée dans le résultat de celui-ci » (Lévy et Lussault cités par Lamara, 2009).

14 individuelles (Lin, 1995), permet de considérer que tout environnement urbain dispose de ses propres ressources, qui n'existent pas en dehors d'une production sociale, individuelle ou collective. Et c'est dans le cadre social et spatial de la migration que nous les identifions. Cette approche nous conduit à traiter le dispositif de la confection en atelier comme une ressource urbaine de la migration contemporaine (quelle que soit l'époque depuis le début du $\mathrm{XX}^{\mathrm{e}}$ siècle), qui a la particularité de se structurer dans le cadre strict des vagues migratoires successives à São Paulo. De sorte que l'atelier de confection est à la fois une ressource de la migration, puisque il est le produit de l'organisation sociale et économique d'une frange de la migration urbaine, et une ressource pour la migration car il forme un marché du travail privilégié pour ces mêmes migrants marginalisés dans l'environnement urbain. L'atelier est cependant une ressource ambivalente, car s'il combine l'offre du logement et de l'emploi, faisant figure de planche de salut pour la migration la plus vulnérable, il est aussi un lieu d'exploitation du travail et de dégradation des conditions de vie des ouvriers et de leur famille.

Enfin, précisons que l'atelier lui-même s'articule sur des ressources urbaines, comme la centralité métropolitaine, dont il dépend et qui le lie étroitement à l'environnement urbain et à différentes modalités d'économies d'agglomération (Cavailhès et al., 2002). 


\section{Les ateliers de confection. Une spécificité urbaine et migratoire}

16 La ville de São Paulo est le centre principal de la production et du commerce de vêtements au Brésil. La partie productive, la confection, repose aujourd'hui sur un nombre croissant d'ateliers ${ }^{7}$ qui sont pour l'essentiel entre les mains d'immigrants internationaux, notamment sud-américains ou sud-coréens. Ces entreprises migratoires, «small imigrant firms ", telles que le sociologue Roger Waldinger (1984) les nomme dans le contexte étasunien, se concentrent en grande partie dans le centre de São Paulo et précisément dans le quart nord-est de cette vaste région de la ville, dans les districts du Brás, du Bom Retiro et du Pari. On les retrouve dans divers types d'endroits, souvent discrètement nichées dans les interstices du bâti valorisé des artères commerçantes, ou en marge de lieux dépréciés et dégradés dont l'usage dominant peut tout aussi bien être commercial, que résidentiel ou industriel.

Les districts du Bom Retiro, Brás et Pari (illustration 1) se constituent au tournant du $\mathrm{XX}^{\mathrm{e}}$ siècle, lorsque São Paulo entre dans une phase de développement industriel et de croissance démographique inédites, portée par une vague migratoire étrangère historique. Ces trois quartiers, qui aujourd'hui font partie du centre-ville, sont alors nouveaux et marginaux ; à la fois, industriels, ouvriers et immigrés. Dès la toute fin du XIX ${ }^{e}$ siècle, E. Reclus en témoigne en décrivant le Brás comme un quartier "peuplé surtout d'Italiens, [qui] s'étend au loin dans la plaine basse et contraste par ses usines, ses rues malpropres, ses égouts vaseux, avec les constructions élégantes et les villas des quartiers occidentaux» (Reclus, 1894, p. 371). Le Bom Retiro est quant à lui associé à l'immigration des Juifs d'Europe de l'Est, il est également un quartier ouvrier, industriel, mais présente un profil moins homogène que le Brás et il compte, par exemple, des institutions scolaires ainsi que des aménagements pour les loisirs en plein air. Le Pari, enfin, se constitue dans le prolongement de la croissance de ces quartiers.

Dès sa formation, l'ensemble constitué des trois districts se distingue par une forte identité fonctionnelle et sociale où prédominent l'habitat ouvrier et le bâti industriel. Les installations industrielles et les cités ouvrières côtoient un bâti hybride, dégradé, où résidences et activités productives s'entremêlent, comme dans les nombreux ateliers qui très vite se multiplient. Au fil des décennies, la désindustrialisation accélère la dégradation de ces quartiers et, même si les vagues migratoires étrangères puis brésiliennes s'y succèdent, la population diminue progressivement à partir de la deuxième moitié du $\mathrm{XX}^{\mathrm{e}}$ siècle, alors que la ville croît à un rythme exceptionnel. Sur le déclin, ils semblent abandonnés et en marge des initiatives très localisées de requalification du centre.

19 S'il est vrai qu'ils contrastent fortement avec le reste de la ville et qu'une image négative leur est associée, ils n'ont pas été totalement désertés, car ce sont encore des quartiers populaires, commerçants, industriels et immigrés. Notamment, l'installation dans les années 1980 d'une migration sud-coréenne dans le Bom Retiro va relancer la filière de l'habillement, en développant à la fois la confection en atelier et le commerce de gros. Contrairement à l'industrie dans son ensemble qui a abandonné les quartiers centraux, les ateliers n'ont jamais disparu du Brás, du Bom Retiro et du Pari, où ils se sont implantés dès les années 1920. Une telle longévité est propre au modèle des ateliers, fondé sur un rapport particulier à l'environnement urbain, entretenu au fil du temps par une succession de vagues migratoires. Car les ateliers de confection, par leur 
taille et en raison d'une articulation du lieu de travail et de l'espace domestique, s'accommodent du bâti industriel comme du résidentiel, qu'ils recyclent à peu de frais en investissant des entrepôts désaffectés, les arrière-cours, les soupentes etc. Le nombre d'ateliers de confection augmente avec l'installation des Coréens qui vont réintroduire la migration internationale au cœur des ateliers, à mesure que la crise des années 1980 impose de lourds changements organisationnels dans l'industrie (soustraitance, flexibilisation) (Buechler, 2014). Les migrants coréens développent alors des compétences dans le design, la production et le commerce de vêtements, en s'appuyant notamment sur des circuits d'importation de tissus de Corée du Sud. Mais progressivement, les entrepreneurs coréens ou d'origine coréenne se spécialisent dans les activités créatives et commerciales, et se délestent de la confection, qu'ils transfèrent à une migration plus récente, venue de Bolivie et du Paraguay8 ${ }^{8}$. Ainsi, s'organise le processus de succession migratoire et l'insertion sur le marché du travail, conformément aux règles énoncées par la queuing theory (Waldinger, 1987) selon laquelle les activités les moins valorisées reviennent aux migrants, et parmi eux, aux derniers venus.

\section{Les enjeux autour de l'offre de logement dans les ateliers}

Pour capter la main-d'œuvre, enjeu essentiel dans une activité à forte intensité de travail, les patrons d'ateliers de confection associent en général au sein du dispositif un espace de production et un lieu de résidence, de sorte que l'insertion des ateliers repose sur un double usage des ressources de l'environnement urbain, à des fins productives et résidentielles. Pour les entrepreneurs de la confection, l'installation dans le centreville, même si elle est avantageuse en termes de coût immobilier et de centralité, est une équation délicate, puisqu'elle nécessite à la fois de connaître et de maîtriser les ressources spécifiques de l'environnement urbain du centre, et de s'adapter aux nombreuses contraintes d'un espace qui, depuis plus d'un demi-siècle, a connu une lente dévalorisation et une dégradation généralisée (illustrations 4 et 5). Dans un contexte de fermeture du marché du travail métropolitain, les ateliers de confection font figure de refuge pour les migrants, qu'ils soient entrepreneurs ou ouvriers, et ce quelles que soient les conditions dans lesquelles ils y évoluent ${ }^{9}$. L'économie urbaine a sa niche économique ${ }^{10}:$ la confection, et son dispositif : l'atelier de confection. 
Illustration 4 - Bâtiment résidentiel où sont installés plusieurs ateliers de confection, Rua Hipódromo et Rua 25 de Abril, quartier du Brás, São Paulo

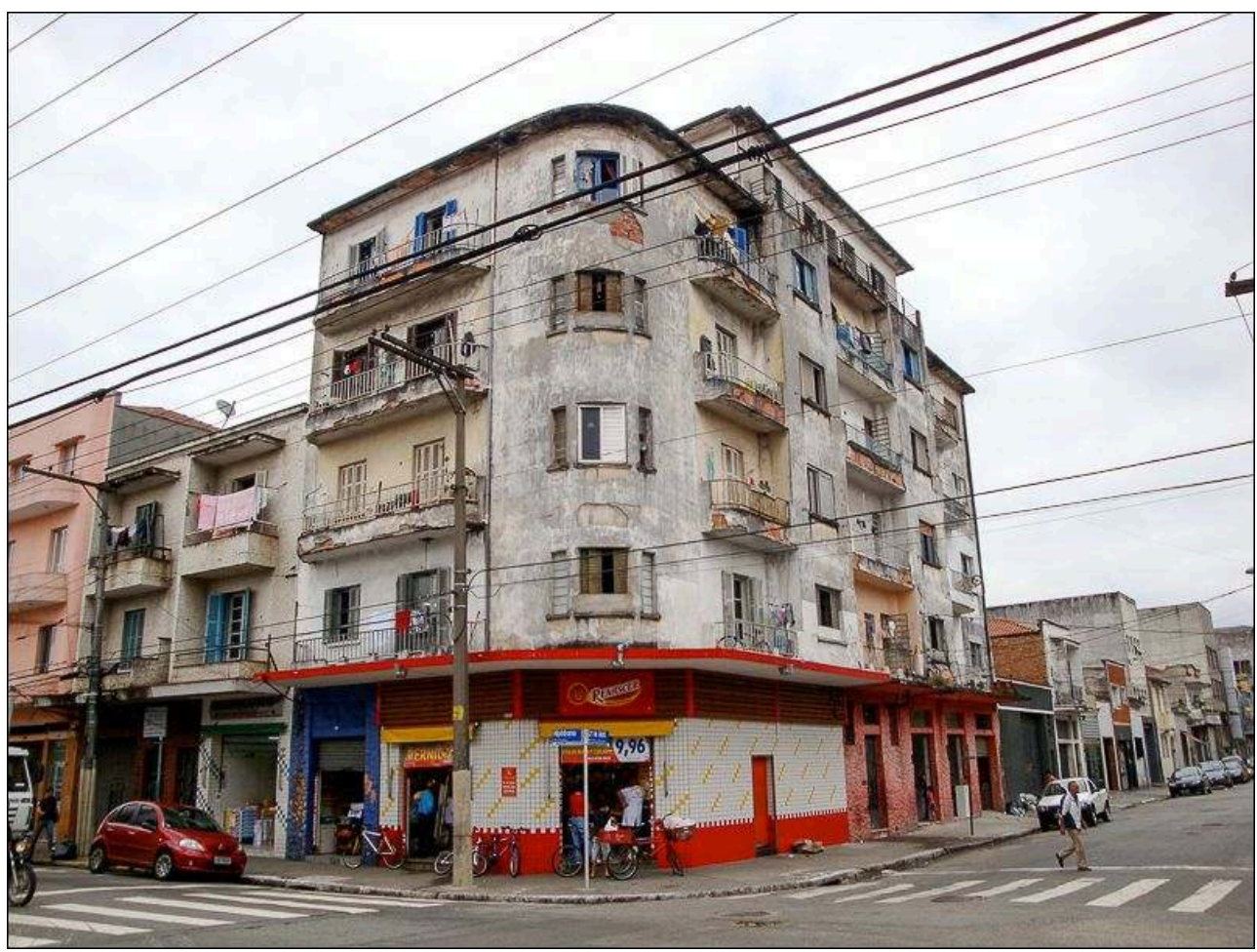

Auteur : S. Souchaud, 22 décembre 2009.

Illustration 5 - Maison abritant un atelier de confection où travaillent et vivent une dizaine d'immigrés Paraguayens, rua dos Italianos, quartier du Bom Retiro à São Paulo

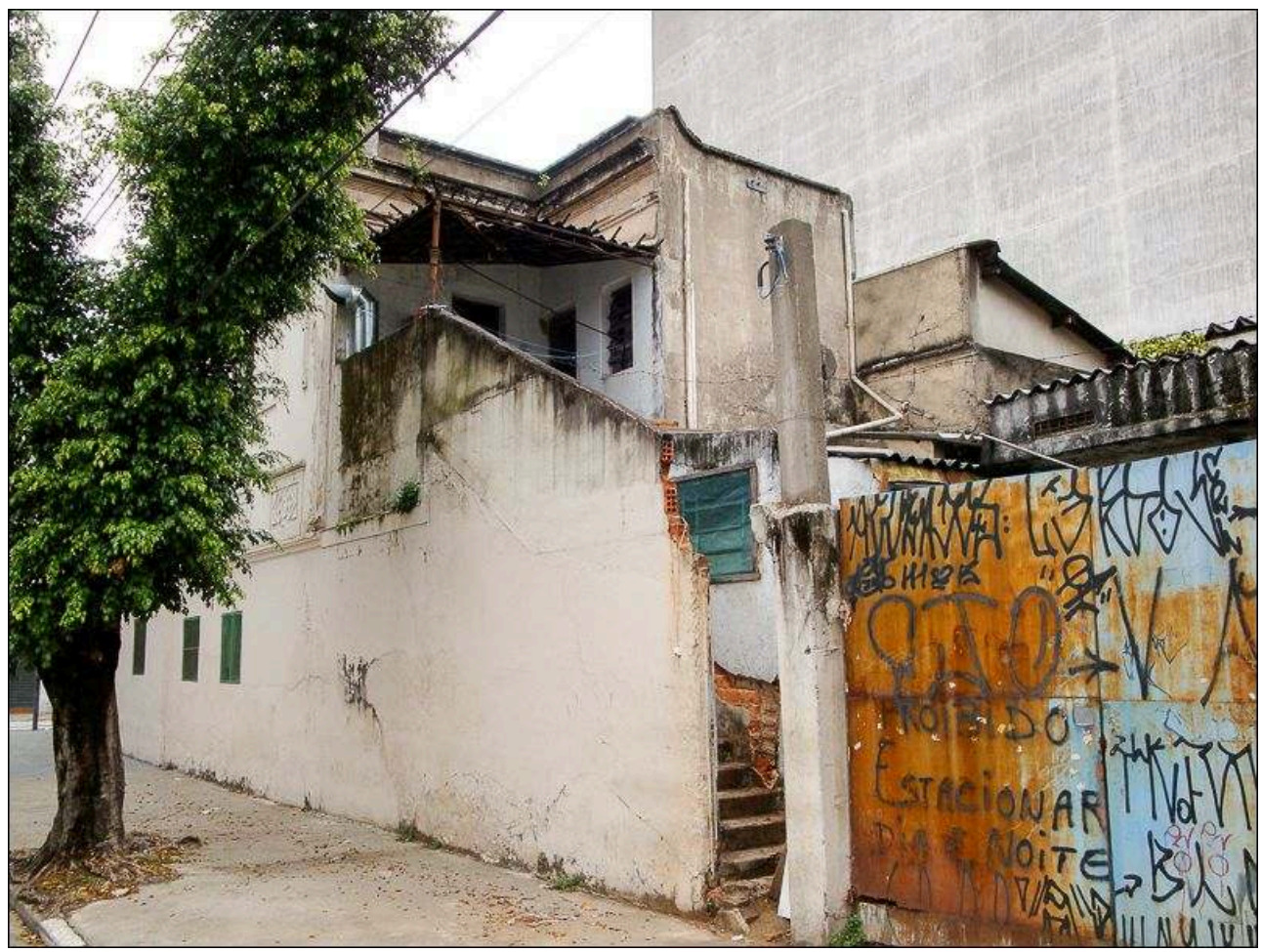

Auteur : S. Souchaud, 9 mai 2010 
Pour fonctionner, ces petits entreprises migratoires («small immigrant firms») s'articulent à des réseaux migratoires mobilisant la main-d'œuvre dans les environnements de proximité, familiaux et de voisinage (villages ou quartiers urbains des espaces de départ comme de destination). Les mécanismes sont bien connus, mais les ateliers sont également confrontés à la difficulté d'attirer et de maintenir les effectifs de main-d'œuvre, car la rotation des ouvriers dans les ateliers est rapide. Elle s'explique tant par les conditions de travail et de salaire, que par l'état de l'offre et la nature des contrats. En effet, dans un secteur où les contrats sont oraux, les droits salariaux négligés, mais où l'offre d'emploi est abondante, les ouvriers circulent d'atelier en atelier, à la recherche de meilleures conditions de travail ou pour sanctionner les abus des patrons. Ceux-ci proposent donc presque systématiquement un logement gratuit aux ouvriers (illustrations 6 et 7), n'ignorant pas qu'ils ont toutes les peines du monde à trouver un hébergement. Les ateliers de São Paulo sont donc des structures qui associent couramment un lieu de travail et un lieu de résidence. Au vu de cette caractéristique, l'on comprend que l'enjeu de l'espace est crucial pour l'existence d'un atelier: il faut un espace de production, un lieu de résidence et de surcroît s'installer de préférence dans le centre de la ville, au cœur de la zone de la confection.

Illustration 6 - Chambre double, dans un appartement collectif hébergeant des ouvriers situé à quelques rues de l'atelier qui les emploie, quartier du Bom Retiro à São Paulo

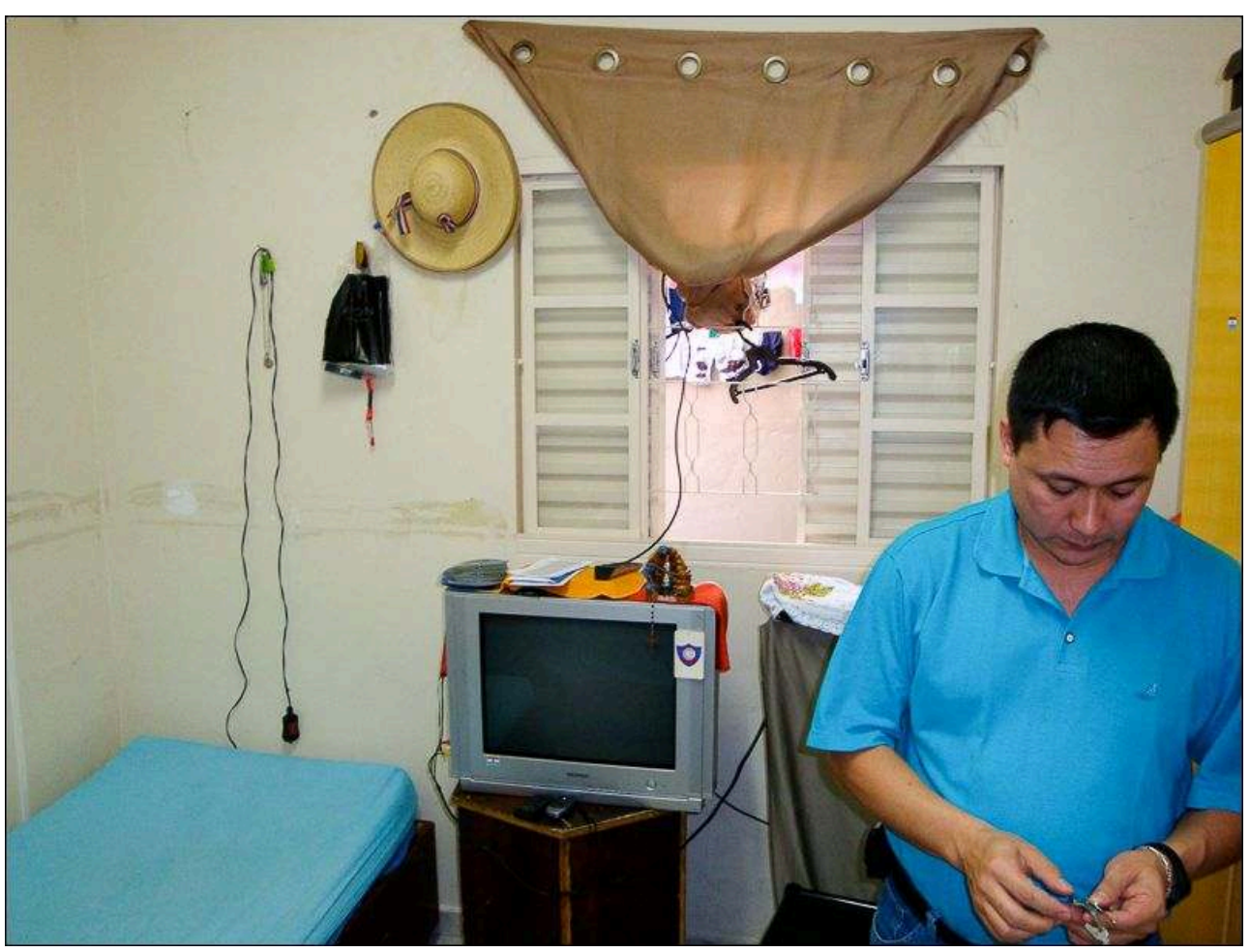

Auteur : S. Souchaud, 27 avril 2010. 
Illustration 7 - Dortoir d'ouvriers, séparé de l'atelier par une cloison, quartier du Bom Retiro, São Paulo

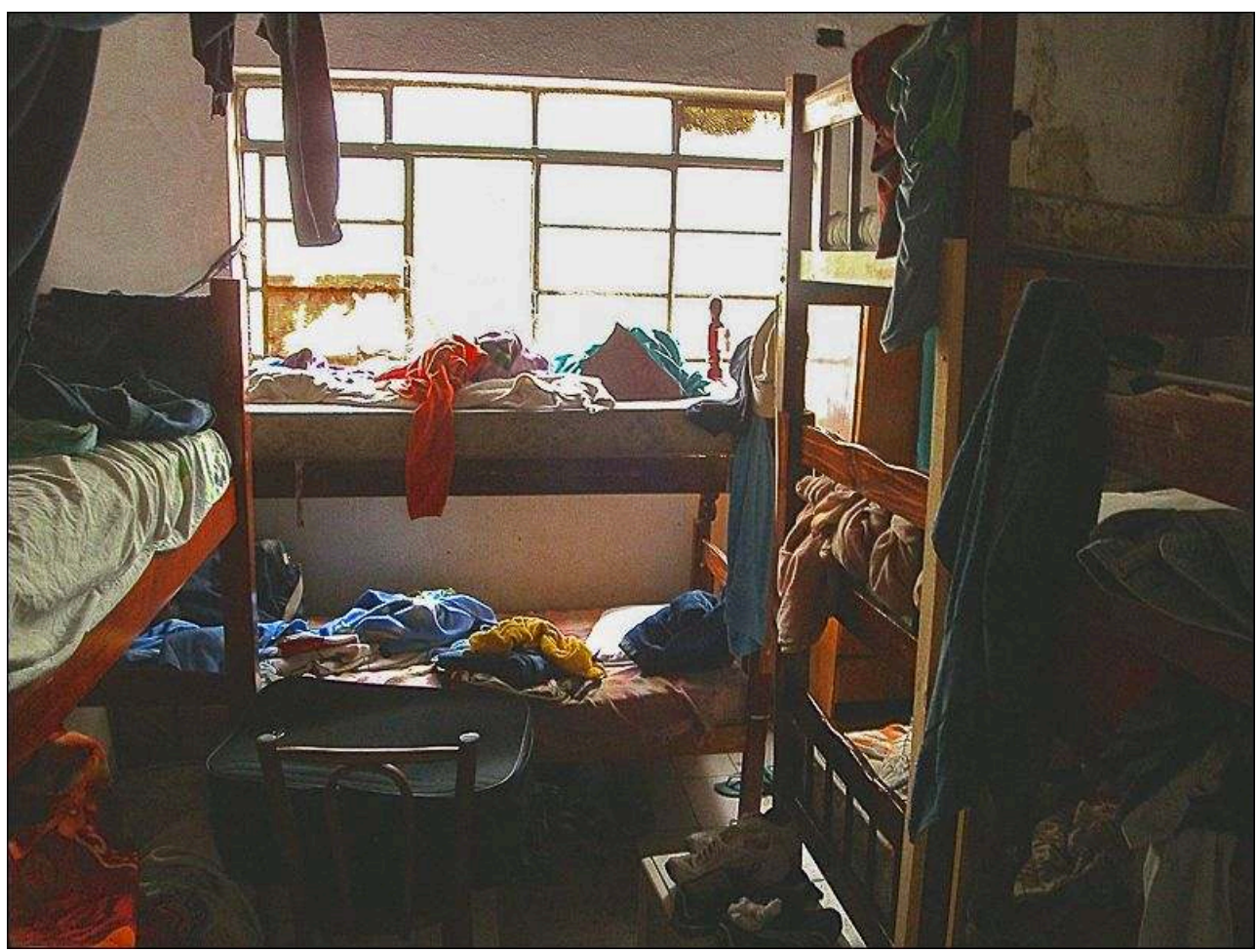

Auteur : S. Souchaud, 9 mai 2010

\section{Rester au centre, s'approprier le bâti pour proposer le logement aux migrants}

Un très grand nombre d'ateliers sont installés dans le cœur de la ville, et surtout dans un espace situé à cheval sur les districts de Brás, Bom Retiro et Pari. Ce quartier est aujourd'hui spécialisé dans le commerce de gros du vêtement; il est le principal marché de vêtements du pays (illustration 1). L'activité s'y déploie jour et nuit, dans la rue, dans les boutiques et les innombrables centres commerciaux spécialisés. Le marché draine, par effet d'agglomération, toutes sortes d'activités liées au vêtement, à son commerce comme à sa production: consommateurs, fournisseurs et donneurs d'ouvrages se côtoient et attirent à leur tour quantité de services et d'activités spécifiques. Cette effervescence générée par la recherche d'économies d'agglomération est le principe de la centralité (Green, 2003).

Les ateliers sont aussi très nombreux dans le secteur. Il est en effet impératif pour eux d'entretenir la proximité avec la demande, car dans la confection, celle-ci évolue rapidement et constamment (Montagné-Villette). De même, il est important d'avoir accès à une offre dense et variée de fournisseurs en concurrence et donc bon marché ; de côtoyer les citadins, d'observer leurs goûts, de sonder leurs attentes, en somme d'être en prise avec l'environnement urbain et sa demande en constante évolution. A São Paulo comme ailleurs, la confection est une industrie intra-urbaine, qui se définit et s'entretient au contact de la culture urbaine, dans laquelle elle puise la dynamique de son existence (Simmel, 1957). 
Cependant, s'il est vital pour les ateliers de s'installer dans le centre-ville, ou d'y être au plus près, rien n'indique que cela soit aisé. Car la concurrence est rude dans cette partie de la ville où les places sont chères - et ce, même si comme dans les métropoles américaines le centre est dégradé - et où l'installation pose de nombreuses difficultés, en raison même de la lente désorganisation et de l'inadaptation fonctionnelle de ces espaces laissés en marge de l'évolution urbaine.

Mais les ateliers ont su s'adapter aux caractéristiques du tissu urbain du centre pour s'y implanter, faisant de certaines contraintes ou obstacles une ressource spécifique. En effet, bon nombre d'ateliers, à des degrés divers, se sont appropriés le bâti du centreville laissé à l'abandon, parce qu'il est bon marché et facilement accessible (illustrations 4 et 5). Grâce à cette offre immobilière déclassée, les ateliers de confection essaiment : les patrons d'ateliers investissent les logements inoccupés, les dépendances de bâtiments commerciaux qui ne sont plus aux normes, ou difficilement accessibles. Une partie des résidences insalubres ou des locaux inadaptés à l'évolution des activités économiques ont donc été recyclés par la confection. Les immigrés, généralement locataires, y ont installé leur activité, le plus souvent illégalement. Par conséquent, certaines configurations du bâti, exclues du circuit économique formel, deviennent une ressource dans le contexte migratoire.

Nous y reviendrons, mais notons d'ores et déjà que les conditions de travail dans ces ateliers implantés dans des locaux dégradés, sombres, exigus, mal ventilés, sont souvent épouvantables, à l'image même des lieux et de leur statut. Et ce contexte entretient la clandestinité de la main-d'œuvre étrangère, car les ateliers qui font l'objet de ces arrangements sont en général nichés dans les interstices les plus inattendus de lieux que l'on croit de prime abord inoccupés. Nous avons ainsi visité des ateliers dans des fonds de cours d'habitations insalubres, des locaux commerciaux abandonnés, un hall d'un appartement exigu, pièces sombres et peu accessibles, et même à l'entresol d'un parking en étage.

27 L'atelier se définit donc en fonction de son rapport au tissu urbain. Sa localisation l'inscrit non seulement dans un usage spécifique des ressources urbaines propres à chaque type d'espace, mais aussi dans un projet entrepreneurial particulier, l'un étant lié à l'autre ${ }^{11}$.

le centre, l'existence d'un excédent d'offre immobilière facilite la présence des ateliers de confection, ou tout au moins retarde leur départ ${ }^{12}$. Cette offre abondante et bon marché permet également aux patrons d'ateliers d'héberger leurs ouvriers, solution qui convient aux patrons comme aux ouvriers.

Grâce à l'offre d'hébergement, les patrons limitent la rotation de la main-d'œuvre et renforcent son encadrement. La main-d'œuvre est l'enjeu central de l'économie des ateliers de confection; il faut trouver des ouvriers, il faut les garder et s'assurer qu'ils se soumettront à une rude discipline, ce qui est assez difficile compte tenu des médiocres conditions de travail, de l'absence de contrat et de l'abondance des offres d'emploi dans les ateliers concurrents. Tous ces éléments contribuent à la dégradation $\mathrm{du}$ travail, alimentent la défiance de part et d'autre, facilitent les renvois abusifs comme les départs précipités. Stabiliser le travail tout en entretenant une forte précarité est l'équation cynique qui fonde le pari relevé par les patrons d'atelier. Pour ce faire, ceux-ci proposent à leurs ouvriers un hébergement sur place ou à proximité du lieu de travail. Les employés sont logés dans des appartements collectifs, où les conditions de vies sont très variables et tout au mieux modestes (illustrations 5 et 6). 
Les ouvriers ne quittent donc pas l'univers de l'atelier, ne perdent pas de temps dans les transports, et se consacrent pleinement au travail, d'autant qu'ils sont payés à la pièce. Pour eux, l'offre d'hébergement résout l'une des principales difficultés de la migration à São Paulo, où se loger pose de nombreux problèmes qui ne sont pas seulement financiers.

D'ordinaire, les études consacrées à la confection à São Paulo présentent l'hébergement sur place des ouvriers des ateliers comme un fait relevant du seul mécanisme du travail sous contrainte, par lequel les patrons usent de l'argument trompeur d'une dette inextinguible que l'ouvrier hébergé contracte auprès de l'hébergeur/employeur dès lors que, selon ceux-ci, la valeur du travail de l'ouvrier serait inférieure au coût de l'hébergement, auquel s'ajoute celui des frais de migration, parfois pris en charge par le patron. Ces formes de travail dit esclave, relevant du mécanisme de la servitude pour dette, sont fréquentes, surtout parmi les jeunes migrants récents qui sont facilement pris au piège. Mais elles seules ne suffisent pas à décrire le système de l'hébergement dans l'économie des ateliers de confection. Car tous les migrants ne sont pas pris au piège de la servitude pour dette, et pour nombre d'entre eux, l'hébergement constitue une ressource non négligeable dans un environnement urbain très fermé, quand l'on ne peut se prévaloir ni d'une expérience professionnelle ni d'un diplôme supérieur. Elles traduisent également une forme de vulnérabilité des patrons et soulignent les tensions en chaîne que l'économie de la confection et le modèle des ateliers entretiennent ${ }^{13}$.

31 Les migrants de la confection ont donc très largement recours à l'hébergement mis à disposition par les patrons. Pour le vérifier et mesurer la pénétration de ce système dans l'économie de la confection et le mode de vie de migrants, nous disposons des informations du recensement de la population de 2010 relatives au temps de déplacement entre le lieu de travail et le lieu de résidence (illustration 8). L'information recouvre différentes situations de résidence sur le lieu de travail et de travail à domicile et, dans le cas particulier des migrants, la configuration la plus fréquente correspond à un hébergement sur le lieu de travail ou à proximité (incluant la catégorie "moins de 5 minutes» mais aussi les valeurs basses de la catégorie «de 5 à 30 minutes »). Alors que dans l'ensemble, les habitants de São Paulo (RMSP) sont peu nombreux à déclarer travailler à domicile $(24,6 \%)$, la résidence sur le lieu de travail, signalée par la catégorie "travail à domicile ", est une pratique majoritaire parmi les migrants paraguayens et boliviens (respectivement 69,6 et $76,1 \%$ ), ceux-là même qui forment l'essentiel des effectifs ouvriers des ateliers. Elle est nettement moins fréquente au sein de la population coréenne, elle aussi traditionnellement impliquée dans la confection. Cela s'explique par l'ancienneté de la migration coréenne et par sa trajectoire dans la confection: les Coréens, encore très présents dans le secteur, sont aujourd'hui rarement ouvriers; ils sont plus souvent patrons d'atelier ou propriétaires de marques de vêtements. Cette trajectoire économique combinant ascension sociale et concentration sectorielle de cette population implique également qu'elle se concentre très fortement dans le quartier du Bom Retiro, à fois quartier de travail et de résidence.

Illustration 8 - Population résidente dans le Belém, Bom Retiro, Brás, Pari, née en Bolivie, au Paraguay et en Corée et travaillant dans la confection, selon le temps de déplacement résidencetravail, en 2010

Pays de naissance 


\begin{tabular}{|l|l|l|l|l|}
\hline Temps de parcours résidence travail & Bolivie & Corée & Paraguay & Total \\
\hline Travail à domicile & 76,1 & 15,1 & 69,6 & 65,8 \\
\hline Moins de 5 mn & 8,3 & 3,7 & 10,1 & 7,8 \\
\hline De 6 à $30 \mathrm{mn}$ & 13,6 & 75,5 & 15,4 & 23,4 \\
\hline De $30 \mathrm{mn}$ à $1 \mathrm{~h}$ & 0,0 & 5,8 & 0,0 & 0,9 \\
\hline Plus d'1 h & 1,0 & 0,0 & 5,0 & 1,3 \\
\hline Sans information & 1,1 & 0,0 & 0,0 & 0,0 \\
\hline Total & 100,0 & 100,0 & 100,0 & 100,0 \\
\hline
\end{tabular}

\section{Conclusion : I'informalité et la vulnérabilité comme principes de l'atelier de confection}

Explorer la notion de ressources urbaines enrichit l'analyse conjointe de la migration et de la production de l'espace urbain, en situant l'analyse sur les dynamiques intraurbaines, pour en relever les détails comme les contradictions. En effet, le modèle d'organisation des ateliers de confection met en évidence que les ressources urbaines relèvent tout autant d'un usage que d'une production de l'espace urbain. Il s'agit d'usage quand les patrons d'ateliers s'approprient et recyclent le bâti dévalorisé et dégradé pour implanter leur activité dans le centre-ville et ainsi bénéficier des avantages compétitifs incomparables de la centralité. Il s'agit de production lorsqu'ils organisent l'hébergement de la main-d'œuvre dans les ateliers : les ateliers sont alors une ressource urbaine pour les migrants démunis, exclus des marchés du travail et du logement. D'une certaine manière, les ateliers comblent ainsi l'insuffisance de l'accès aux ressources dont pâtissent les migrants récents, et favorisent leur insertion urbaine. Réciproquement, le dispositif de l'atelier permet de capter la main-d'œuvre immigrée, confinée dans l'espace social et économique de la métropole.

Cependant, le recours à ces ressources a un coût social élevé ${ }^{14}$, parce qu'il entretient plus qu'il ne solutionne l'état de vulnérabilité des migrants les plus démunis. Plus exactement, lorsqu'elle produit des ressources urbaines spécifiques, l'économie de la confection transforme à son bénéfice cet état de vulnérabilité en situation de dépendance. De plus, pour s'approprier les ressources foncières négligées par le marché immobilier et se fondre dans le centre, les ateliers s'adaptent aux contraintes de l'architecture du quartier. L'insertion dans un bâti compact, morcelé et ancien, en tous points inapproprié au regard des dispositions réglementaires qui encadrent et régissent l'activité industrielle et le logement, entretient et aggrave l'informalité qui prévaut dans le secteur.

Par conséquent, les ateliers de confection du centre-ville sont à l'image du bâti où ils se nichent, et leur organisation ressemble fort aux structures qui les hébergent. Insérés 
dans un environnement dégradé, remodelé au fil des cycles économiques et urbains, les ateliers sont pour la plupart des espaces de travail dangereux et des lieux de vie insalubres. Dans l'économie métropolitaine de la confection, les ressources de l'environnement urbain alimentent une économie de la misère.

\section{BIBLIOGRAPHY}

Bastia T., 2007. From Mining to Garment Workshops: Bolivian Migrants in Buenos Aires. Journal of Ethnic and Migration Studies, 33 (4), p. 655-669.

Bonacich E., Appelbaum R.-P., 2000. Behind the label. Inequality in the Los Angeles Apparel Industry. Los Angeles, University of California Press, 395 p.

Bredeloup S., Pliez O., 2005. Migrations entre les deux rives du Sahara. Autrepart, 36, p. 3-20.

Buechler S.-J., 2014. Labor in a Globalizing City. Economic Restructuring in São Paulo, Brazil. New York, Springer, $340 \mathrm{p}$.

Cavailhès J., Frankhauser P., Peeters D., Thomas I., 2002. Aménités urbaines et périurbaines dans une aire métropolitaine de forme fractale. Revue d'Économie Régionale \& Urbaine, 5, p. 729-760.

Choi K.-J., 1991. Além do arco-íris: a imigração coreana no Brasil. São Paulo, Dissertação de mestrado USP.

Green N.-L., 2003. Fashion, Flexible Specialization, and the Sweatshop. A historical problem. In D.E. Bender, R.-A. Greenwald (Eds.), Sweatshop USA: The American Sweatshop in Historical and Global Perspective, New York, Routledge, p. 37-55.

IBGE,2012. Censo demográfico 2010. Microdados da Amostra; Rio de Janeiro, FIBGE.

Kontic B., 2007. Inovação e redes sociais: A indústria da moda em São Paulo. Doutorado, departamento de sociologia, USP, São Paulo, 157 p.

Lamara H., 2009. Les deux piliers de la construction territoriale : coordination des acteurs et ressources territoriales. Développement durable et territoires, p. 1-20.

Leite M. de P., 2004. Tecendo a precarização: trabalho a domicílio e estratégias sindicais na indústria de confecção em São Paulo. Trabalho, Educação e Saúde, 2 (1), p. 239-275.

Light I., 2002. Immigrant Place Entrepreneurs in Los Angeles, 1970-99. International Journal of Urban and Regional Research, 26 (2), p. 215-228.

Lin N., 1995. Les ressources sociales : une théorie du capital social. Revue française de sociologie, 36 (4), p. 685-704.

Ma Mung E., 1999. La dispersion comme ressource, Cultures \& Conflits, (33-34), p. 89-103.

Massey D.-S., Arango J., Hugo G., Kouaouci A., Pellegrin A., Taylor E.-J., 1998. Worlds in Motion. Understanding International Migration at the End of the Millennium. Oxford, Clarendon Press, $376 \mathrm{p}$.

Mera C., 2012. Los migrantes coreanos en la industria textil de la Ciudad de Buenos Aires. Inserción económica e identidades urbanas. Revue Européenne des Migrations Internationales, 28 (4), p. 67-87. 
Monbeig P., 1953a. La croissance de São Paulo. Revue de géographie alpine, 41 (1), p. 59-97.

Monbeig, P., 1953b. La croissance de São Paulo (suite et fin). Revue de géographie alpine, 41 (2), p. 261-309.

Montagné-Villette S., 1990. Le Sentier. Un espace ambigu. Paris, Masson, 140 p.

Piché V., 2013. Les fondements des théories migratoires contemporaines. In Victor Piché (ed.), Les théories de la migration, Paris, Ined éditions, p. 15-60.

Pochmann M. (ed)., 2004. Reestruturação Produtiva: Perspectivas de Desenvolvimento Local com Incluão Social. Rio de Janeiro, Vozes, $350 \mathrm{p}$.

Portes A., 1995. Economic Sociology and the Sociology of Immigration: a Conceptual Overview. In Alejandro Portes (Ed.), The Economic Sociology of Immigration. Essays on Networks, Ethnicity, and Entrepreneurship, p. 1-41.

Reclus E., 1894. Nouvelle géographie universelle. La terre et les hommes. Amérique du Sud. L'Amazonie et La Plata. Paris, Hachette, 834 p.

Silva C. Freire da, 2008. Trabalho informal e redes de subcontratação: dinâmicas urbanas da indústria de confecção em São Paulo. dissertação de mestrado, departamento de sociologia, Universidade de São Paulo, São Paulo, 141 p.

Simmel G., 1957. Fashion. The American Journal of Sociology, 62 (6), p. 541-558.

Souchaud S., 2012. A confecção : nicho étnico ou nicho econômico para a imigração latinoamericana em São Paulo? In Rosana Baeninger (Ed.), Imigração boliviana no Brasil, Campinas, Nepo/ Unicamp, Fapesp, CNPq, Unfpa, p. 75-92 http://www.nepo.unicamp.br/publicacoes/ _bolivianos.html.

Souchaud S. 2014. Localisation et organisation de l'industrie de la confection à São Paulo, Brésil. Autrepart, 1 (69), p. 131-151.

Vallée J., Commenges H., Perchoux C., Kestens Y., Chaix B., 2015. Mobilité quotidienne et accès potentiel aux ressources urbaines : quelles inégalités sociales? In Marie-Flore Mattei, Denise Pumain (eds.), Données urbaines 7, Paris, Economica, p. 87-95.

Waldinger R., 1984. Immigrant Enterprise in the New York Garment Industry. Social Problems, 32 (1), p. 60-71.

Waldinger R., 1987. Immigrants and Minorities: Conflict in the Job Markets Dissent, 34 (4), p. 519-522.

Waldinger R., 1990. Ethnicity and Entrepreneurship. Annual Review of Sociology (16), p. 111-135.

Waldinger R., 1994. The Making of an Immigrant Niche. International Migration Review, 28 (1), p. 3-30.

Waldinger R., Lapp M., 1993. Back to the Sweatshop or Ahead to the Informal Sector? International Journal of Urban and Regional Research, 17 (1), p. 6-29.

\section{NOTES}

1. La région Nord-Est du Brésil (Nordeste) est composée de huit États : Alagoas, Bahia, Ceará, Maranhão, Paraíba, Pernambuco, Piauí, Rio Grande do Norte, Sergipe. Elle rassemble environ le quart de la population brésilienne et elle est un foyer d'émigration vers la ville São Paulo depuis près d'un siècle. 
2. Il n'existe pas d'évaluation fiable du nombre d'ateliers de confection à São Paulo. Ces structures sont méconnues. De même, il est très difficile d'évaluer l'importance numérique de la migration bolivienne et paraguayenne dans la confection en atelier. Cependant, nous pensons qu'en raison des changements économiques et sociaux intervenus dans les milieux populaires à São Paulo depuis une quinzaine d'années, la confection en atelier a été délaissée par ces catégories de population, notamment les femmes issues de la migration interne. Ces recompositions du marché du marché du travail métropolitain, et notamment la brèche ouverte dans l'économie de la confection, sont à l'origine de la croissance de la migration internationale. La migration internationale récente occupe donc aujourd'hui (et à nouveau) une place centrale dans confection en ateliers de la région métropolitaine (RMSP).

3. Le centre de São Paulo regroupe 10 districts. Selon le recensement, en 2010, la population du centre atteint 478000 individus, dont 34000 dans le Bom Retiro, 29000 dans la Brás et 17000 dans le Pari. La région métropolitaine de São Paulo (RMSP), constituée 39 municípios, compte près de 20 millions d'habitants. La population du município São Paulo, au cœur de la métropole, en rassemble environ 11 millions.

4. L'auteur remercie les relecteurs anonymes dont les analyses, assorties de recommandations précises, ont beaucoup aidé à la préparation de ce texte.

5. Au sens large de système de relations sociales dans lesquels s'inscrivent les comportements individuels.

6. Recensement localisé de 2,2 millions d'équipements en France (Vallée et al., 2015).

7. Nous n'avons pas connaissance d'une estimation fiable du nombre de ces ateliers. Mais l'augmentation de l'immigration régionale (notamment bolivienne et paraguayenne), sa concentration sectorielle et géographique (dans la confection et à São Paulo) sont des éléments qui confirment les informations recueillies lors de notre enquête qui font état d'une croissance du nombre d'ateliers à São Paulo.

8. Un processus semblable de succession migratoire dans la confection, de la migration coréenne à la migration bolivienne et paraguayenne, est survenu à Buenos Aires. Il a été analysé par C. Mera (2012).

9. Les entrepreneurs ont le plus souvent débuté comme simple ouvrier. La condition de patron recouvre une grande diversité de statuts et de situations, mais globalement les patrons d'atelier sont de petits entrepreneurs à la tête d'entreprises instables et précaires. La formation d'un entreprenariat étranger dans des activités à faible coût d'entrée (comme la confection) est révélateur des grandes difficultés que connaissent ces populations sur le marché du travail. La surreprésentation des étrangers dans l'entreprenariat doit être interprétée comme la conséquence des contraintes du marché du travail local et non comme une prédisposition culturelle (Green, 2013).

10. Nous qualifions l'économie de l'atelier de niche économique et non de niche ethnique, car nous relevons peu d'éléments proprement ethniques dans l'atelier de confection, où d'ailleurs les vagues migratoires, internes et internationales, se sont succédé depuis le début $\mathrm{du} \mathrm{XX}^{\mathrm{e}}$ siècle. L'atelier de confection est davantage l'expression de la marginalisation de groupes minorisés dans la société urbaine que de la communalisation de ces groupes.

11. Dans les quartiers périphériques, les ateliers et les projets des patrons qui les dirigent se définissent dans un rapport au foncier différent. On y trouve notamment des entrepreneurs débutants, qui ont commencé leur carrière dans la confection, comme ouvrier, dans le centre et qui, au fil du temps et des étapes résidentielles et professionnelles, ont accumulé les compétences nécessaires à la constitution d'un atelier. Il est important de souligner que le projet entrepreneurial en banlieue est associé à un projet d'accession à la propriété résidentielle, ce qui constitue une énorme différence avec les ateliers du centre. Les migrants, en couple, ou à plusieurs (souvent d'une même fratrie), achètent une parcelle, y construisent leur habitation où ils intègrent un atelier (comprenant l'espace de travail et le lieu de résidence des ouvriers). Ces 
constructions neuves sont l'aboutissement d'une trajectoire urbaine et professionnelle marquée par la réussite sociale.

12. Le taux d'inoccupation du parc résidentiel dans ce secteur du centre-ville s'élevait à des taux record pour la métropole dans les années 1990.

13. Il va sans dire que la vulnérabilité des patrons, quand elle existe, se situe à l'égard des donneurs d'ordre et non des ouvriers; elle n'institue donc pas une forme d'équilibre dans la relation entre l'ouvrier et le patron, ni même une possibilité de réparation du premier vis-à-vis du second dans un rapport où le patron reste largement maître du jeu.

14. Débat autour de l'appréciation de la valeur sociale de la confection que l'on retrouve concernant le commerce ambulant informel.

\section{ABSTRACTS}

This article aims to analyze the production and access to urban resources in a context of migration. Urban resources are treated as devices and practices that are constitutive of the urban environment. To characterize them, we target groups of South American migrants, workers and small employers inserted into the sweatshop economy. We will see how, in a metropolitan context where international migrants face difficulties entering the labor market and access to housing, workshops are organized by re-using urban fabric elements, namely certain frame configurations, and produce their own supply of housing to labor, to guarantee its cheapness and productivity. Therefore, workshops system is a user as much as a producer of specific urban resources.

Cet article a pour objectif l'analyse de la production et de l'accès aux ressources urbaines dans un contexte migratoire. Les ressources urbaines sont considérées comme les dispositifs et les pratiques constitutives de l'environnement urbain. Pour les caractériser, nous ciblons des groupes de travailleurs immigrés sud-américains, ouvriers et petits patrons insérés dans les ateliers de confection qui sont des dispositifs où se cristallisent, entre accès et production, différentes façons d'articuler les ressources urbaines, selon les statuts sociaux-économiques et l'insertion dans l'environnement. Ainsi, nous verrons comment, dans le contexte métropolitain de São Paulo, les patrons d'ateliers se réapproprient le bâti dévalorisé du centre-ville pour y organiser leurs ateliers lesquels, afin de capter une main-d'œuvre marginalisée sur les marchés du travail et du logement, mêlent l'hébergement à l'offre d'activité. L'analyse des ressources urbaines, de leur usage (réaffectation de bâtiments dévalorisés à des fins économiques) et de leur production (offre de travail assortie d'une offre de logement) éclaire la spécificité sociale et économique des ateliers de confection et nous renseigne sur leur insertion métropolitaine.

\section{INDEX}

Mots-clés: migration internationale, industrie de la confection, ressource urbaine, São Paulo, Brésil

Keywords: International migration, Garment Industry, Urban Resources, São Paulo, Brazil Subjects: Sur le Champ - Sur le Terrain 


\section{AUTHOR}

\section{SYLVAIN SOUCHAUD}

Sylvain Souchaud, sylvain.souchaud@gmail.com, est Chargé de recherche à l'Institut de recherche pour le développement (IRD), membre de l'UMR Migrations et société IRD-Université Paris Diderot-CNRS. 